\title{
One-loop effective action of QCD at high temperature using the heat kernel method'
}

\author{
E. Megías \\ Departamento de Física Moderna, Universidad de Granada. 18071-Granada (Spain)
}

\begin{abstract}
Perturbation theory is an important tool to describe the properties of QCD at very high temperatures. Recently a new technique has been proposed to compute the one-loop effective action of QCD at finite temperature by making a gauge covariant derivative expansion, which is fully consistent with topologically small and large gauge transformations (also time dependent transformations) [1]. This technique is based on the heat kernel expansion, and the thermal Wilson line plays an essential role [2]. We consider a general $\mathrm{SU}\left(N_{c}\right)$ gauge group.
\end{abstract}

Introduction. The effective action (EA) plays a prominent role in quantum field theory, since it embodies the renormalized properties of the system. To one loop it takes the form $c \operatorname{Tr} \log (K)$, where $K$ is the differential operator controlling the quadratic quantum fluctuations above a classical background. This quantity is afflicted by ultraviolet divergences, and it is useful to express it by means of a proper time representation [3],

$$
-\operatorname{Tr} \log (K)=\int_{0}^{\infty} \frac{d \tau}{\tau} \operatorname{Tr} e^{-\tau K}=\int_{0}^{\infty} \frac{d \tau}{\tau} \int d^{D} x \operatorname{tr}\left\langle x\left|e^{-\tau K}\right| x\right\rangle .
$$

"tr" refers to trace in internal spaces. The (diagonal) heat kernel $\left\langle x\left|e^{-\tau K}\right| x\right\rangle$ is UV finite.

The heat kernel at finite temperature has been computed for a Klein-Gordon operator, ${ }^{2}$ through the so called heat kernel expansion, in the completely general case of non Abelian and non stationary gauge fields and external fields [1, 2]. It is of the form

$$
\left\langle x\left|e^{-\tau\left(M-D_{\mu}^{2}\right)}\right| x\right\rangle=(4 \pi \tau)^{-D / 2} \sum_{n} a_{n}^{T}(x) \tau^{n}
$$

It is an expansion in local and gauge covariant operators. The so called Seeley-DeWitt coefficients, $a_{n}^{T}$, are contructed with operators of mass dimesion $2 n$. The untraced Polyakov loop or thermal Wilson line plays a fundamental role in maintaining manifest gauge invariance at each order. It appears inside $a_{n}^{T}$, and it is defined as

$$
\Omega(x)=T \exp \left(i \mathrm{~g} \int_{x_{0}}^{x_{0}+\beta} A_{0}\left(x_{0}^{\prime}, \mathbf{x}\right) d x_{0}^{\prime}\right) .
$$

\footnotetext{
${ }^{1}$ Presented at the IX Hadron Physics and VII Relativistic Aspects of Nuclear Physics, Angra dos Reis, Rio de Janeiro, Brazil, March 28 to April 03, 2004.

${ }^{2}$ We consider Klein-Gordon operators of the form, $K=M(x)-D_{\mu}^{2}$, where $D_{\mu}=\partial_{\mu}-\operatorname{ig} A_{\mu}(x)$ is the covariant derivative, $M(x)$ is a scalar (external) field, and $A_{\mu}(x)$ are the gauge fields.
} 
Effective action of QCD. In Ref. [1] the heat kernel has been applied to obtain the 1-loop EA of QCD at high temperature. The Euclidean partition function of QCD is

$$
Z[A, \bar{q}, q]=\int \mathscr{D} A \prod_{\alpha=1}^{N_{f}} \mathscr{D} \bar{q}_{\alpha} \mathscr{D} q_{\alpha} \exp \left\{-\frac{1}{2} \int d^{4} x \operatorname{tr}\left(F_{\mu \nu}^{2}\right)-\int d^{4} x \sum_{\alpha=1}^{N_{f}} \bar{q}_{\alpha} i \not D q_{\alpha}\right\}
$$

where $F_{\mu \nu}=i \mathrm{~g}^{-1}\left[D_{\mu}, D_{v}\right]$ is the field strength. The gauge group of color is $\operatorname{SU}\left(N_{c}\right)$.

The full EA can be obtained upon functional integration of the quark and gluon fields. The quark contribution to the EA at 1-loop is [convention $\left.Z=e^{-\Gamma[A]}\right]$

$$
\Gamma_{q}[A]=-\frac{N_{f}}{2} \operatorname{Tr} \log \left(-\not \not^{2}\right)=: \int d^{4} x \mathscr{L}_{q}(x) .
$$

For the gluon fields we use the background field method, in which the gauge field is split into a classical background field plus a quantum fluctuation, i.e. $A_{\mu} \rightarrow A_{\mu}+a_{\mu}$, in (4). The standard procedure consists of adding a gauge fixing term and the corresponding Fadded-Popov term in the action. The gluon contribution is then

$$
\Gamma_{g}[A]=\frac{1}{2} \operatorname{Tr} \log \left(-\delta_{\mu v} \widehat{D}_{\lambda}^{2}+2 i g \widehat{F}_{\mu \nu}\right)-\operatorname{Tr} \log \left(-\widehat{D}_{\mu}^{2}\right)=: \int d^{4} x \mathscr{L}_{g}(x)
$$

The operators in (5) and (6) act in the fundamental and adjoint representations, respectively. They are of the Klein-Gordon form, so we can use the proper time representation. ${ }^{3}$ For the different orders of the heat kernel expansion, we obtain

$$
\begin{aligned}
\mathscr{L}_{0}(x)= & \frac{\pi^{2} T^{4}}{3}\left(\frac{1+2 N_{c} N_{f}-N_{c}^{2}}{15}-\frac{N_{f}}{4} \operatorname{tr}\left[\left(1-4 \bar{v}^{2}\right)^{2}\right]+2 \widehat{\operatorname{tr}}\left[\widehat{v}^{2}(1-\widehat{v})^{2}\right]\right) \\
\mathscr{L}_{2}(x)= & \left(\frac{1}{2}-\mathrm{g}^{2} \beta_{0} \log (\mu / 4 \pi T)-\frac{\mathrm{g}^{2} N_{c}}{6(4 \pi)^{2}}\right) \operatorname{tr}\left(F_{\mu v}^{2}\right) \\
& +\frac{11 \mathrm{~g}^{2}}{12(4 \pi)^{2}} \widehat{\operatorname{tr}}\left[(\psi(\widehat{v})+\psi(1-\widehat{v})) \widehat{F}_{\mu \nu}^{2}\right] \\
& -\frac{\mathrm{g}^{2} N_{f}}{3(4 \pi)^{2}} \operatorname{tr}\left[\left(\psi\left(\frac{1}{2}+\bar{v}\right)+\psi\left(\frac{1}{2}-\bar{v}\right)\right) F_{\mu \nu}^{2}\right]+\frac{2 \mathrm{~g}^{2}}{3(4 \pi)^{2}}\left(N_{c}-N_{f}\right) \operatorname{tr}\left[E_{i}^{2}\right] \\
\mathscr{L}_{3}(x)= & -\frac{2 \mathrm{~g}^{2}}{(4 \pi)^{4}} \frac{N_{f}}{T^{2}} \operatorname{tr}\left[( \psi ^ { \prime \prime } ( \frac { 1 } { 2 } + \overline { v } ) + \psi ^ { \prime \prime } ( \frac { 1 } { 2 } - \overline { v } ) ) \left(\frac{1}{60}\left[D_{\mu}, F_{\mu v}\right]^{2}-\frac{1}{24}\left[D_{\lambda}, F_{\mu \nu}\right]^{2}\right.\right. \\
& \left.\left.+\frac{8}{45} i \mathrm{~g} F_{\mu \nu} F_{v \lambda} F_{\lambda \mu}-\frac{1}{20}\left[D_{0}, F_{\mu v}\right]^{2}+\frac{1}{30}\left[D_{i}, E_{i}\right]^{2}+\frac{1}{15} i \mathrm{~g} E_{i} F_{i j} E_{j}\right)\right] \\
& +\frac{\mathrm{g}^{2}}{2(4 \pi)^{4}} \frac{1}{T^{2}} \widehat{\operatorname{tr}}\left[( \psi ^ { \prime \prime } ( \widehat { v } ) + \psi ^ { \prime \prime } ( 1 - \widehat { v } ) ) \left(\frac{1}{30}\left[\widehat{D}_{\mu}, \widehat{F}_{\mu \nu}\right]^{2}-\frac{1}{3}\left[\widehat{D}_{\lambda}, \widehat{F}_{\mu \nu}\right]^{2}\right.\right. \\
& \left.\left.+\frac{61}{45} i \mathrm{~g} \widehat{F}_{\mu \nu} \widehat{F}_{v \lambda} \widehat{F}_{\lambda \mu}-\frac{3}{5}\left[\widehat{D}_{0}, \widehat{F}_{\mu v}\right]^{2}+\frac{1}{15}\left[\widehat{D}_{i}, \widehat{E}_{i}\right]^{2}+\frac{2}{15} i \mathrm{~g} \widehat{E}_{i} \widehat{F}_{i j} \widehat{E}_{j}\right)\right]
\end{aligned}
$$

\footnotetext{
3 The integrals over $\tau$ are one-valued functions of the Polyakov loop, so gauge invariance is manifest.
} 
where $-\frac{1}{2}<\bar{v}<\frac{1}{2}$ and $0<\widehat{v}<1 .{ }^{4} \psi(q)$ is the digamma function. $E_{i}=F_{0 i}$ is the electric field. $\mathrm{g}$ is the running coupling defined in the $\overline{\mathrm{MS}}$ scheme. $\beta_{0}=\left(11 N_{c}-2 N_{f}\right) /\left(3(4 \pi)^{2}\right)$.

A new technique has been proposed recently for 1-loop QCD at high temperature [4]. It goes beyond ours in that all orders in $A_{0}$ are retained. But the authors use stationary configurations. Our results for $\mathscr{L}_{2}$ agree for some terms that can be directly compared.

Dimensional reduced effective theory. In the high temperature limit non stationary fluctuations become heavy and are therefore suppressed, and QCD behaves as an effective three-dimensional theory for the stationary configurations only. This effective theory is obtained by i) using stationary backgrounds and ii) taking purely non-stationary fluctuations only, that is, removing the static Matsubara mode. Doing this, one obtains ${ }^{5}$

$$
\begin{aligned}
\mathscr{L}_{0}^{\prime}(\mathbf{x})= & \mathrm{g}^{2}\left(\frac{N_{c}}{3}+\frac{N_{f}}{6}\right) T\left\langle A_{0}^{2}\right\rangle+\frac{\mathrm{g}^{4}}{4 \pi^{2} T}\left\langle A_{0}^{2}\right\rangle^{2}+\frac{\mathrm{g}^{4}}{12 \pi^{2} T}\left(N_{c}-N_{f}\right)\left\langle A_{0}^{4}\right\rangle, \\
\mathscr{L}_{4}^{\prime}(\mathbf{x})= & \frac{1}{2 T}\left\langle F_{\mu \nu}^{2}\right\rangle, \\
\mathscr{L}_{6}^{\prime}(\mathbf{x})= & -\frac{2}{15} \frac{\mathrm{g}^{2} \zeta(3)}{(4 \pi)^{4} T^{3}}\left[i \mathrm{~g}\left(\frac{2}{3} N_{c}-\frac{14}{3} N_{f}\right)\left\langle F_{\mu \nu} F_{v \lambda} F_{\lambda \mu}\right\rangle-\left(10 N_{c}-28 N_{f}\right)\left\langle\left[D_{\mu}, F_{\mu \nu}\right]^{2}\right\rangle\right. \\
& -\left(18 N_{c}-21 N_{f}\right)\left\langle\left[D_{0}, F_{\mu \nu}\right]^{2}\right\rangle+\left(2 N_{c}-14 N_{f}\right)\left\langle\left[D_{i}, E_{i}\right]^{2}\right\rangle+110 \mathrm{~g}^{2}\left\langle A_{0}^{2}\right\rangle\left\langle F_{\mu \nu}^{2}\right\rangle \\
& \left.+i g\left(4 N_{c}-28 N_{f}\right)\left\langle E_{i} F_{i j} E_{j}\right\rangle+\mathrm{g}^{2}\left(110 N_{c}-140 N_{f}\right)\left\langle A_{0}^{2} F_{\mu \nu}^{2}\right\rangle+220 \mathrm{~g}^{2}\left\langle A_{0} F_{\mu \nu}\right\rangle^{2}\right]
\end{aligned}
$$

where $\langle X\rangle:=\operatorname{tr}(X)$, and $B_{i}=\frac{1}{2} \varepsilon_{i i k} F_{j k}$ is the magnetic field. $\mathscr{L}_{6}^{\prime}$ has been computed in [5] for the gluon sector, and in [6] for the quark sector and for SU(3) in the absence of chromomagnetic field $\left(A_{i}=0\right)$. Our results agree with these.

Work done in collaboration with E. Ruiz Arriola and L. L. Salcedo. Supported by funds provided by the Spanish DGI and FEDER Grant No. BFM2002-03218, Junta de Andalucía Grant No. FQM-225, and EURIDICE Contract No. HPRN-CT-2002-00311.

\section{REFERENCES}

1. E. Megías, E. Ruiz Arriola and L. L. Salcedo, Phys. Rev. D in press, arXiv:hep-ph/0312133

2. E. Megías, E. Ruiz Arriola and L. L. Salcedo, Phys. Lett. B 563, 173 (2003).

3. J. S. Schwinger, Phys. Rev. 82, 664 (1951).

4. D. Diakonov and M. Oswald, Phys. Rev. D 68, 025012 (2003), and arXiv:hep-ph/0312126

5. S. Chapman, Phys. Rev. D 505308 (1994).

6. J. Wirstam, Phys. Rev. D 65, 014020 (2002).

${ }^{4} \Omega(x)=e^{2 \pi i \bar{v}}$ is in the fundamental representation, and $\widehat{\Omega}(x)=e^{2 \pi i \widehat{v}}$ is in the adjoint one.

${ }^{5}$ In that formulas we have rescaled $A_{i}$ and $A_{0}$ with different renormalization factors, so that $\mathscr{L}_{4}^{\prime}$ looks like the zero temperature renormalized tree level: $\mathrm{g} \rightarrow Z_{g}^{-1 / 2} \mathrm{~g}, A_{i} \rightarrow Z_{M}^{1 / 2} A_{i}, A_{0} \rightarrow Z_{E}^{1 / 2} A_{0}$; with $Z_{M}=Z_{g}=1+2 \mathrm{~g}^{2} \beta_{0}\left[\log (\mu / 4 \pi T)+\gamma_{E}\right]-\frac{\mathrm{g}^{2}}{3(4 \pi)^{2}}\left[-N_{c}+8 N_{f} \log 2\right], \quad Z_{E}=Z_{M}-\frac{2 \mathrm{~g}^{2}}{3(4 \pi)^{2}}\left(N_{c}-N_{f}\right)$. 